Open J. Math. Anal., Vol. 2(2018), No. 2, pp. 129 - 141

Website: https://pisrt.org/psr-press/journals/oma/

ISSN: 2616-8111 (Online) 2616-8103 (Print)

http://dx.doi.org/10.30538/psrp-oma2018.0023

\title{
ANALYTIC STUDY ON HILFER FRACTIONAL LANGEVIN EQUATIONS WITH IMPULSES
}

\author{
S. HARIKRISHNAN ${ }^{1}$, E. M. ELSAYED, K. KANAGARAJAN
}

\begin{abstract}
In this paper, we find a solution of a new type of Langevin equation involving Hilfer fractional derivatives with impulsive effect. We formulate sufficient conditions for the existence and uniqueness of solutions. Moreover, we present Hyers-Ulam stability results.
\end{abstract}

Mathematics Subject Classification: 26A33, 34K40, 34K14.

Key words and phrases: Langevin equation; impulsive condition; fixed point theorem; Ulam stability.

\section{Introduction}

Fractional differential equation (FDEs) has gained increasing attention because of their varied applications in applied sciences and engineering, see the monograph [1, 2, 3. The memory and hereditary of various material and process can be properly described as FDEs. Due to their importance and necessity of FDEs many researchers focused their work towards existence theory and stability criteria. In this work, we study existence of solution for FDEs with Hilfer fractional derivative (HFD) which was initiated by Hilfer [1]. HFD interpolate both classical Riemann-Liouville (RL) and the Liouville-Caputo (LC) fractional derivatives. Recently, HFD is studied in many papers for detailed study, see 4, 5, 6, 7, 8, 9, 10.

In 1908, Langevin introduced a concept of an equation of motion of a Brownian particle which is named after Langevin equation. Langevin equations have been

Received 19-11-218. Accepted 01-12-2018.

1 Corresponding Author

(C) 2018 S. Harikrishnan, E. M. Elsayed, K. Kanagarajan. This is an open access article distributed under the Creative Commons Attribution License, which permits unrestricted use, distribution, and reproduction in any medium, provided the original work is properly cited. 
widely used to describe stochastic differential equation 11. For systems in complex media, standard Langevin equation does not provide the correct description of the dynamics. As a result, various generalizations of Langevin equations have been offered to describe dynamical processes in a fractal medium. One such generalization is the generalized Langevin equation which incorporates the fractal and memory properties with a dissipative memory kernel into the Langevin equation. These give a rise to the Langevin equation involving fractional order. In 2007, Fa [12 discussed variance and velocity correlation of Langevin equations with both RL and LC fractional derivative. In 2011, Existence of solutions is analysed in [13. Since them many authors discussed existence of solution with different conditions, see [14, 15, 16, 17.

Impulsive differential equations have been focused since it serves as an important tool to characterize the phenomena in which sudden, discontinuous jumps occur in various fields of science and engineering, and impulsive FDEs have received many attentions, see [18, 19, 20].

The concept of stability for a functional equation arises when we replace the functional equation by an inequality which acts as a perturbation of the equation. Considerable attention has been given to the study of the Ulam-Hyers (UH) and Ulam-Hyers-Rassias(UHR) stability. More details from historical point of view and recent developments of such stabilities are reported in $21,22,23,24,25,26]$. Consider the following system of Langevin differential equation involving HFD with impulse effect

$$
\left\{\begin{array}{l}
D^{\alpha_{1}, \beta}\left(D^{\alpha_{2}, \beta}+\lambda\right) x(t)=f(t, x(t)), \quad t \in J^{\prime}:=J \backslash\left\{t_{1}, t_{2}, \ldots, t_{m}\right\} \\
J=[a, b], \quad t \notin t_{k}, \\
\left.\Delta I^{1-\gamma} x(t)\right|_{t=t_{k}}=\psi_{k}\left(x\left(t_{k}\right)\right), \quad t=t_{k}, \quad k=1,2, \ldots, m \\
\left.I^{1-\gamma} x(t)\right|_{t=a}=x_{a}, \quad \gamma=\left(\alpha_{1}+\alpha_{2}\right)(1-\beta)+\beta
\end{array}\right.
$$

where $D^{\alpha_{1}, \beta}, D^{\alpha_{2}, \beta}\left(0<\left(\alpha_{1}, \alpha_{2}\right)<1,0 \leq \beta \leq 1\right)$ are the GRL fractional derivative of orders $\alpha_{1}, \alpha_{2}$ and type $\beta$. Here, the function $f: J \times R \rightarrow R$ is continuous, $I_{k}: R \rightarrow R$, and $a \in R, a=t_{0}<t_{1}<\ldots<t_{m}<t_{m+1}=b$, $\left.\Delta I^{1-\gamma} x(t)\right|_{t=t_{k}}=I^{1-\gamma} x\left(t_{k}^{+}\right)-I^{1-\gamma} x\left(t_{k}^{-}\right), I^{1-\gamma} x\left(t_{k}^{+}\right)=\lim _{h \rightarrow 0+} x\left(t_{k}+h\right)$ and $I^{1-\gamma} x\left(t_{k}^{-}\right)=\lim _{h \rightarrow 0-} x\left(t_{k}+h\right)$ represent the right and left limits of $x(t)$ at $t=t_{k}$.

The existence and uniqueness results for the problem (1), some of the following conditions have to be satisfied;

(H1) Let $f: J \times R \rightarrow R$ be a continuous function and there exists a positive constant $L_{f}>0$, such that

$$
|f(t, x)-f(t, y)| \leq L_{f}|x-y|, \text { for all } x, y \in R .
$$

(H2) Let the functions $\psi_{k}: R \rightarrow R$ are continuous and there exists a constant $L_{\psi}>0$, such that

$$
\left|\psi_{k}(x)-\psi_{k}(y)\right| \leq L_{\psi}|x-y| \text {, for all } x, y \in R, k=1,2, \ldots, m \text {. }
$$


(H3) There exists an increasing finctions $\varphi \in P C_{1-\gamma}(J, R)$ and there exists $\lambda_{\varphi}>0$ such that for any $t \in J$

$$
I^{\alpha} \varphi(t) \leq \lambda_{\varphi} \varphi(t) .
$$

The paper is organized as follows: In Section 2, we present some necessary definitions and preliminary results that will be used to prove our main results. The proofs of our main results are given in Section 3.

\section{Preliminaries}

In this section, we present some known definition and results that help us in proving of our main results. Consider the following space

$$
P C(J, R)=\left\{x: J \rightarrow R: x(t) \in C\left(t_{k}, t_{k+1}\right], k=0, \ldots, m ;\right\}
$$

there exists $x\left(t_{k}^{+}\right)$and $x\left(t_{k}^{-}\right)$. Now we consider the weighted space $P C_{\gamma}(J, R)$,

$$
P C_{\gamma}(J, R)=\left\{x:\left.\left(t-t_{k}\right)^{\gamma} x(t)\right|_{t \in\left[t_{k}, t_{k+1}\right]} \in C\left[t_{k}, t_{k+1}\right], k=0, \ldots, m\right\},
$$

where $0 \leq \gamma<1$, which is a Banach space with norm

$$
\|x\|_{P C_{\gamma}}=\sup _{t \in\left(t_{k}, t_{k+1}\right]}\left\{\left(t-t_{k}\right)^{\gamma} x(t)\right\}, k=0, \ldots, m .
$$

Definition 2.1. 2 The Riemann-Liouville (RL) fractional integral of order $\alpha>0$ of function $f:[0, \infty) \rightarrow R$ can be written as

$$
I^{\alpha} f(t)=\frac{1}{\Gamma(\alpha)} \int_{0}^{t}(t-s)^{\alpha-1} f(s) d s .
$$

Definition 2.2. 2 The RL fractional derivative of order $\alpha>0$ of a continuous function $f:[0, \infty) \rightarrow R$ can be written as

$$
D^{\alpha} f(t)=\frac{1}{\Gamma(n-\alpha)}\left(\frac{d}{d t}\right)^{n} \int_{0}^{t}(t-s)^{\alpha-n+1} f(s) d s,
$$

provided that the right side is pointwise defined on $[0, \infty)$.

Definition 2.3. 2] The LC fractional derivative of order $\alpha>0$ of a continuous function $f:[0, \infty) \rightarrow R$ can be written as

$$
{ }^{C} D^{\alpha} f(t)=D^{\alpha}\left[f(t)-\sum_{k=0}^{n-1} \frac{t^{k}}{k !} f^{k}(0)\right], t>0, n-1<\alpha<n .
$$

Definition 2.4. [1] The HFD of order $0<\alpha<1$ and $0 \leq \beta \leq 1$ of function $f(t)$ is defined by

$$
D^{\alpha, \beta} f(t)=\left(I^{\beta(1-\alpha)} D\left(I^{(1-\beta)(1-\alpha)} f\right)\right)(t) .
$$

The HFD is considered as an interpolation between the RL and LC fractional derivative and the relations are given below. 
Remark 2.5. (i) Operator $D^{\alpha, \beta}$ also can be written as

$$
D^{\alpha, \beta}=\left(I^{\beta(1-\alpha)} D\left(I^{(1-\beta)(1-\alpha)}\right)\right)=I^{\beta(1-\alpha)} D^{\gamma}, \quad \gamma=\alpha+\beta-\alpha \beta .
$$

(ii) If $\beta=0$, then $D^{\alpha, \beta}=D^{\alpha, 0}$ is called RL fractional derivative.

(iii) If $\beta=1$, then $D^{\alpha, \beta}=I^{1-\alpha} D$ is called LC fractional derivative.

Lemma 2.6. 10] If $\alpha>0$ and $\beta>0$, then there exists

$$
\left[I^{\alpha}(t)^{\beta-1}\right](x)=\frac{\Gamma(\beta)}{\Gamma(\beta+\alpha)} x^{\beta+\alpha-1},
$$

and

$$
\left[D^{\alpha}(t)^{\alpha-1}\right](x)=0, \quad 0<\alpha<1 .
$$

Lemma 2.7. 10. If $\alpha>0$ and $\beta>0$ and $f \in L^{1}(a, b]$, then there exists the following properties

$$
I^{\alpha} I^{\beta} f(t)=I^{\alpha+\beta} f(t)
$$

and

$$
D^{\alpha} I^{\alpha} f(t)=f(t) .
$$

Next, we shall give the definitions and the criteria of UH stability and UHR stability for Langevin differential equations with impulsive effect by GRL fractional derivative. Let $\epsilon$ be a positive number and $\varphi: J \rightarrow R^{+}$be a continuous function, for every $t \in J^{\prime}$ and $k=1,2, \ldots, m$, we have the following inequalities

$$
\begin{gathered}
\begin{cases}\left|D^{\alpha_{1}, \beta}\left(D^{\alpha_{2}, \beta}+\lambda\right) z(t)-f(t, z(t))\right| & \leq \epsilon, \\
\left.\Delta I^{1-\gamma} z(t)\right|_{t=t_{k}}-\psi_{k}\left(z\left(t_{k}\right)\right) \mid & \leq \epsilon,\end{cases} \\
\begin{cases}\left|D^{\alpha_{1}, \beta}\left(D^{\alpha_{2}, \beta}+\lambda\right) z(t)-f(t, z(t))\right| & \leq \epsilon \varphi(t), \\
\left.\Delta I^{1-\gamma} z(t)\right|_{t=t_{k}}-\psi_{k}\left(z\left(t_{k}\right)\right) \mid & \leq \epsilon \varphi(t),\end{cases} \\
\begin{cases}\left|D^{\alpha_{1}, \beta}\left(D^{\alpha_{2}, \beta}+\lambda\right) z(t)-f(t, z(t))\right| & \leq \varphi(t), \\
\left.\Delta I^{1-\gamma} z(t)\right|_{t=t_{k}}-\psi_{k}\left(z\left(t_{k}\right)\right) \mid & \leq \varphi(t),\end{cases}
\end{gathered}
$$

Definition 2.8. The system equations given in (1) is UH stable if there exists a real number $C_{f}>0$ such that for each $\epsilon>0$ and for each solution $z \epsilon$ $P C_{1-\gamma}(J, R)$ of the inequality (2) there exists a solution $x \in P C_{1-\gamma}(J, R)$ of Eq. (1) with

$$
|z(t)-x(t)| \leq C_{f} \epsilon, \quad t \in J .
$$

Definition 2.9. The system equations given in (1) is generalized UH stable if there exist $\varphi \in P C_{1-\gamma}\left(J, R^{+}\right), \varphi_{f}(0)=0$ such that for each solution $z \in$ $P C_{1-\gamma}(J, R)$ of the inequality (2) there exists a solution $x \in P C_{1-\gamma}(J, R)$ of Eq. (1) with

$$
|z(t)-x(t)| \leq \varphi_{f} \epsilon, \quad t \in J
$$


Definition 2.10. The system equations given in (1) is UHR stable with respect to $\varphi \in P C_{1-\gamma}\left(J, R^{+}\right)$if there exists a real number $C_{f}>0$ such that for each solution $z \in P C_{1-\gamma}(J, R)$ of the inequality (3) there exists a solution $x \in P C_{1-\gamma}(J, R)$ of Eq. (1) with

$$
|z(t)-x(t)| \leq C_{f} \epsilon \varphi(t), \quad t \in J .
$$

Definition 2.11. The system equations given in (1) is generalized UHR stable with respect to $\varphi \in P C_{1-\gamma^{+}}(J, R)$ if there exists a real number $C_{f, \varphi}>0$ such that for each solution $z \in P C_{1-\gamma}(J, R)$ of the inequality (4) there exists a solution $x \in P C_{1-\gamma}(J, R)$ of Eq. (1) with

$$
|z(t)-x(t)| \leq C_{f, \varphi} \varphi(t), \quad t \in J .
$$

Remark 2.12. A function $z \in P C_{1-\gamma}(J, R)$ is a solution of the inequality

$$
\left|D^{\alpha_{1}, \beta}\left(D^{\alpha_{2}, \beta}+\lambda\right) z(t)-f(t, z(t))\right| \leq \epsilon,
$$

if and only if there exist a function $g \in P C_{1-\gamma}(J, R)$ and a sequence $g_{k}, k=$ $1,2, \ldots, m$ (which depend on $z$ ) such that

(i) $|g(t)| \leq \epsilon,\left|g_{k}\right|<\epsilon$.

(ii) $D^{\alpha_{1}, \beta}\left(D^{\alpha_{2}, \beta}+\lambda\right) z(t)=f(t, z(t))+g(t)$.

(iii) $\left.\Delta I^{1-\gamma} z(t)\right|_{t_{k}}=\psi_{k}\left(z\left(t_{k}\right)\right)+g_{k}$.

Lemma 2.13. 27. Let $a(t)$ be a nonnegative function locally integrable on $a \leq$ $t<b$ for some $b \leq \infty$, and let $g(t)$ be a nonnegative, nondecreasing continuous function defined on $a \leq t<b$, such that $g(t) \leq K$ for some constant $K$. Further let $x(t)$ be a nonnegative locally integrable on $a \leq t<b$ function satisfying

$$
|x(t)| \leq a(t)+g(t) \int_{a}^{t}(t-s)^{\alpha-1} x(s) d s, \quad t \in[a, b)
$$

with some $\alpha>0$. Then

$$
|x(t)| \leq a(t)+\int_{a}^{t}\left[\sum_{n=1}^{\infty} \frac{(g(t) \Gamma(\alpha))^{n}}{\Gamma(n \alpha)}(t-s)^{n \alpha-1}\right] a(s) d s, \quad a \leq t<b .
$$

Remark 2.14. Under the hypethesis of Lemma 2.13 let $a(t)$ be a nondecreasing function on $[0, T)$. Then $y(t) \leq a(t) E_{\alpha}\left(g(t) \Gamma(\alpha) t^{\alpha}\right)$, where $E_{\alpha}$ is the MittagLeffler function defined by

$$
E_{\alpha}(z)=\sum_{k=0}^{\infty} \frac{z^{k}}{\Gamma(k \alpha+1)}, z \in C, \operatorname{Re}(\alpha)>0 .
$$

Lemma 2.15. 26] Let $x \in P C_{1-\gamma}(J, R)$ satisfies the following inequality

$$
|x(t)| \leq c_{1}+c_{2} \int_{0}^{t}(t-s)^{\alpha-1}|x(t)| d s+\sum_{0<t_{k}<t} \psi_{k}\left|x\left(t_{k}\right)\right|,
$$

where $c_{1}$ is a nonnegative, continuous and nondecreasing function and $c_{2}, \psi_{i}$ are constants. Then

$$
|x(t)| \leq c_{1}\left(1+\psi E_{\alpha}\left(c_{2} \Gamma(\alpha) t^{\alpha}\right)^{k} E_{\alpha}\left(c_{2} \Gamma(\alpha) t^{\alpha}\right) \text { for } t \in\left(t_{k} . t_{k+1}\right],\right.
$$


where $\psi=\sup \left\{\psi_{k}: k=1,2,3, \ldots, m\right\}$.

Theorem 2.16. 28 (Schauder Fixed Point Theorem) Let $E$ be a Banach space and $Q$ be a nonempty bounded convex and closed subset of $E$ and $N: Q \rightarrow Q$ is compact, and continuous map. Then $N$ has at least one fixed point in $Q$.

Theorem 2.17. 28 (Banach Fixed Point Theorem) Suppose $Q$ be a nonempty closed subset of a Banach space $E$. Then any contraction mapping $N$ from $Q$ into itself has a unique fixed point.

\section{Main results}

In this section, we study the main results on the existence of solution for Equation (1). We need the following Lemma to establish our main results.

Lemma 3.1. Let $f: J \times R \rightarrow R$ be continuous. A function $x$ is a solution of the fractional integral equation

$$
x(t)=\left\{\begin{array}{l}
\frac{x_{a}}{\Gamma(\gamma)}(t-a)^{\gamma-1}-\lambda I_{a}^{\alpha_{2}} x(t)+I_{a}^{\alpha_{1}+\alpha_{2}} f(t, x(t)) \quad \text { if } \quad t \in\left[a, t_{1}\right], \\
\frac{\left(t-t_{k}\right)^{\gamma-1}}{\Gamma(\gamma)}\left[x_{a}+\sum_{0<t_{k}<t} \psi_{k}\left(x\left(t_{k}\right)\right)-\sum_{0<t_{k}<t} \lambda I_{t_{k-1}}^{\left(1-\alpha_{1}\right)(1-\beta)+\alpha_{2} \beta} x\left(t_{k}\right)\right. \\
\left.+\sum_{0<t_{k}<t} I_{t_{k-1}}^{1+\beta\left(\alpha_{1}+\alpha_{2}-1\right)} f\left(t_{k}, x\left(t_{k}\right)\right)\right]-\lambda I_{t_{k}}^{\alpha_{2}} x(t) \\
+I_{t_{k}}^{\alpha_{1}+\alpha_{2}} f(t, x(t)) \quad \text { if } \quad t \in\left(t_{k}, t_{k+1}\right],
\end{array}\right.
$$

where $k=1, \ldots, m$, if and only if $x$ is a solution of the fractional initial value problem

$$
\begin{aligned}
D^{\alpha_{1}, \beta}\left(D^{\alpha_{2}, \beta}+\lambda\right) x(t) & =f(t, x(t)), \\
\left.\Delta I^{1-\gamma} x(t)\right|_{t=t_{k}} & =\psi_{k}\left(x\left(t_{k}\right)\right), \\
I^{1-\gamma} x(a) & =x_{a} .
\end{aligned}
$$

Theorem 3.2. Assume that [H1] and [H2] are fulfilled. If

$$
\begin{aligned}
\rho=[ & \frac{1}{\Gamma(\gamma)}\left(m L_{\psi}(b-a)^{\gamma-1}+\frac{m \lambda B\left(\gamma,\left(1-\alpha_{1}\right)(1-\beta)+\alpha_{2} \beta\right)}{\Gamma\left(\left(1-\alpha_{1}\right)(1-\beta)+\alpha_{2} \beta\right)}(b-a)^{1+\alpha_{2}}\right. \\
& \left.+\frac{m L_{f} B\left(\gamma, 1+\beta\left(\alpha_{1}+\alpha_{2}-1\right)\right)}{\Gamma\left(1+\beta\left(\alpha_{1}+\alpha_{2}-1\right)\right)}(b-a)^{\alpha_{1}+\alpha_{2}}\right)+\frac{\lambda B\left(\gamma, \alpha_{2}\right)}{\Gamma\left(\alpha_{2}\right)}(b-a)^{\alpha_{2}} \\
& \left.+\frac{B\left(\gamma, \alpha_{1}+\alpha_{2}\right)}{\Gamma\left(\alpha_{1}+\alpha_{2}\right)}(b-a)^{\alpha_{1}+\alpha_{2}}\right]<1,
\end{aligned}
$$

then the Equation (1) has a unique solution.

Proof. The proof is based on the Banach fixed point theorem. Define the operator $N: P C_{1-\gamma}(J, R) \rightarrow P C_{1-\gamma}(J, R)$. The equivalent integral equation (5) 
which can be written in the operator form as follows

$$
N x(t)=\left\{\begin{array}{l}
\frac{\left(t-t_{k}\right)^{\gamma-1}}{\Gamma(\gamma)}\left[x_{a}+\sum_{0<t_{k}<t} \psi_{k}\left(x\left(t_{k}\right)\right)-\sum_{0<t_{k}<t} \lambda I_{t_{k-1}}^{\left(1-\alpha_{1}\right)(1-\beta)+\alpha_{2} \beta} x\left(t_{k}\right)\right. \\
\left.+\sum_{0<t_{k}<t} I_{t_{k-1}}^{1+\beta\left(\alpha_{1}+\alpha_{2}-1\right)} f\left(t_{k}, x\left(t_{k}\right)\right)\right]-\lambda I_{t_{k}}^{\alpha_{2}} x(t) \\
+I_{t_{k}}^{\alpha_{1}+\alpha_{2}} f(t, x(t))
\end{array}\right.
$$

First, we show that $N$ maps $B_{r}$ into $B_{r}$. It is clear that $N$ is well defined on $P C_{1-\gamma}(J, R)$. Moreover for any $x \in B_{r}$, we have

$$
\begin{array}{rl}
\mid N & x(t)\left(t-t_{k}\right)^{1-\gamma} \mid \\
\leq & \frac{1}{\Gamma(\gamma)}\left[x_{a}+\sum_{0<t_{k}<t}\left|\psi_{k}\left(x\left(t_{k}\right)\right)\right|+\sum_{0<t_{k}<t} \lambda I_{t_{k-1}}^{\left(1-\alpha_{1}\right)(1-\beta)+\alpha_{2} \beta}\left|x\left(t_{k}\right)\right|\right. \\
& \left.+\sum_{0<t_{k}<t} I_{t_{k-1}}^{1+\beta\left(\alpha_{1}+\alpha_{2}-1\right)}\left|f\left(t_{k}, x\left(t_{k}\right)\right)\right|\right]+\lambda I_{t_{k}}^{\alpha_{2}}|x(t)|+I_{t_{k}}^{\alpha_{1}+\alpha_{2}}|f(t, x(t))| \\
\leq & \frac{1}{\Gamma(\gamma)}\left[x_{a}+\sum_{0<t_{k}<t}\left|\psi_{k}\left(x\left(t_{k}\right)\right)-\psi_{k}(0)\right|+\sum_{0<t_{k}<t}\left|\psi_{k}(0)\right|\right. \\
& +\sum_{0<t_{k}<t} \lambda I_{t_{k-1}}^{\left(1-\alpha_{1}\right)(1-\beta)+\alpha_{2} \beta}\left|x\left(t_{k}\right)\right| \\
& +\sum_{0<t_{k}<t} I_{t_{k-1}}^{1+\beta\left(\alpha_{1}+\alpha_{2}-1\right)}\left|f\left(t_{k}, x\left(t_{k}\right)\right)-f\left(t_{k}, 0\right)\right| \\
& \left.+\sum_{0<t_{k}<t} I_{t_{k-1}}^{1+\beta\left(\alpha_{1}+\alpha_{2}-1\right)}\left|f\left(t_{k}, 0\right)\right|\right] \\
& +\left(t-t_{k}\right)^{1-\gamma} \lambda I_{t_{k}}^{\alpha_{2}}|x(t)|+\left(t-t_{k}\right)^{1-\gamma} I_{t_{k}}^{\alpha_{1}+\alpha_{2}}|f(t, x(t))-f(t, 0)| \\
& +\left(t-t_{k}\right)^{1-\gamma} I_{t_{k}}^{\alpha_{1}+\alpha_{2}}|f(t, 0)| \\
\leq & \frac{1}{\Gamma(\gamma)}\left[x_{a}+m L_{\psi}(b-a)^{\gamma-1}\|x\|_{P C_{1-\gamma}}+m L_{2}\right. \\
& +\frac{m \lambda B\left(\gamma,\left(1-\alpha_{1}\right)(1-\beta)+\alpha_{2} \beta\right)}{\Gamma\left(\left(1-\alpha_{1}\right)(1-\beta)+\alpha_{2} \beta\right)}(b-a)^{1+\alpha_{2}}\|x\|_{P C_{1-\gamma}} \\
& +\frac{m L_{f} B\left(\gamma, 1+\beta\left(\alpha_{1}+\alpha_{2}-1\right)\right)}{\Gamma\left(1+\beta\left(\alpha_{1}+\alpha_{2}-1\right)\right)}(b-a)^{\alpha_{1}+\alpha_{2}}\|x\|_{P C_{1-\gamma}} \\
& +\frac{\lambda B\left(\gamma, \alpha_{2}\right)}{\Gamma\left(\alpha_{2}\right)}(b-a)^{\alpha_{2}}\|x\|_{P C_{1-\gamma}}+\frac{B\left(\gamma, \alpha_{1}+\alpha_{2}\right)}{\Gamma\left(\alpha_{1}+\alpha_{2}\right)}(b-a)^{\alpha_{1}+\alpha_{2}}\|x\|_{P C_{1-\gamma}} \\
& +\alpha_{1} \\
& \left.+(b-a)^{1+\left(\alpha_{1}+\alpha_{2}-1\right) \beta}\right] \\
& \left.\left.+\alpha_{2}-1\right) \beta\right) \\
&
\end{array}
$$




$$
\begin{aligned}
& +\frac{l_{1}}{\Gamma\left(\alpha_{1}+\alpha_{2}+1\right)}(b-a)^{\alpha_{1}+\alpha_{2}-\gamma+1} \\
& \leq r .
\end{aligned}
$$

Consequently $N$ maps $B_{r}$ into itself. Let $x, y \in P C_{1-\gamma}(J, R)$ and $t \in J$, then we have

$$
\begin{aligned}
\mid & (N x(t)-N y(t))\left(t-t_{k}\right)^{1-\gamma} \mid \\
\leq & \frac{1}{\Gamma(\gamma)}\left[\sum_{0<t_{k}<t}\left|\psi_{k}\left(x\left(t_{k}\right)\right)-\psi_{k}\left(y\left(t_{k}\right)\right)\right|+\sum_{0<t_{k}<t} \lambda I_{t_{k-1}}^{\left(1-\alpha_{1}\right)(1-\beta)+\alpha_{2} \beta}\left|x\left(t_{k}\right)-y\left(t_{k}\right)\right|\right. \\
& \left.+\sum_{0<t_{k}<t} I_{t_{k-1}}^{1+\beta\left(\alpha_{1}+\alpha_{2}-1\right)}\left|f\left(t_{k}, x\left(t_{k}\right)\right)-f\left(t_{k}, y\left(t_{k}\right)\right)\right|\right] \\
+ & \left(t-t_{k}\right)^{1-\gamma} \lambda I_{t_{k}}^{\alpha_{2}}|x(t)-y(t)| \\
& +\left(t-t_{k}\right)^{1-\gamma} I_{t_{k}}^{\alpha_{1}+\alpha_{2}}|f(t, x(t))-f(t, y(t))| \\
\leq & \frac{1}{\Gamma(\gamma)}\left[\sum_{0<t_{k}<t} L_{\psi}\left|\left(x\left(t_{k}\right)\right)-\left(y\left(t_{k}\right)\right)\right|+\sum_{0<t_{k}<t} \lambda I_{t_{k-1}}^{\left(1-\alpha_{1}\right)(1-\beta)+\alpha_{2} \beta}\left|x\left(t_{k}\right)-y\left(t_{k}\right)\right|\right. \\
& \left.+\sum_{0<t_{k}<t} I_{t_{k-1}}^{1+\beta\left(\alpha_{1}+\alpha_{2}-1\right)} L_{f}\left|x\left(t_{k}\right)-y\left(t_{k}\right)\right|\right]+\left(t-t_{k}\right)^{1-\gamma} \lambda I_{t_{k}}^{\alpha_{2}}|x(t)-y(t)| \\
& +\left(t-t_{k}\right)^{1-\gamma} I_{t_{k}}^{\alpha_{1}+\alpha_{2}} L_{f}|x(t)-y(t)| .
\end{aligned}
$$

Thus

$$
\begin{aligned}
\| & N x-N y \|_{P C_{1-\gamma}} \\
\leq & {\left[\frac { 1 } { \Gamma ( \gamma ) } \left(m L_{\psi}(b-a)^{\gamma-1}+\frac{m \lambda B\left(\gamma,\left(1-\alpha_{1}\right)(1-\beta)+\alpha_{2} \beta\right)}{\Gamma\left(\left(1-\alpha_{1}\right)(1-\beta)+\alpha_{2} \beta\right)}(b-a)^{1+\alpha_{2}}\right.\right.} \\
& \left.+\frac{m L_{f} B\left(\gamma, 1+\beta\left(\alpha_{1}+\alpha_{2}-1\right)\right)}{\Gamma\left(1+\beta\left(\alpha_{1}+\alpha_{2}-1\right)\right)}(b-a)^{\alpha_{1}+\alpha_{2}}\right)+\frac{\lambda B\left(\gamma, \alpha_{2}\right)}{\Gamma\left(\alpha_{2}\right)}(b-a)^{\alpha_{2}} \\
& \left.+\frac{B\left(\gamma, \alpha_{1}+\alpha_{2}\right)}{\Gamma\left(\alpha_{1}+\alpha_{2}\right)}(b-a)^{\alpha_{1}+\alpha_{2}}\right]\|x-y\|_{P C_{1-\gamma}} \\
= & \rho\|x-y\|_{P C_{1-\gamma}} .
\end{aligned}
$$

This yields that $N$ has unique fixed point which is solution of Equation (1).

Theorem 3.3. Assume that [H1] and [H2] are satisfied. Then, Equation (1) has at least one solution.

Proof. Let us denote $f(t, 0)=l_{1}, \psi_{k}(0)=l_{2}$. Consider,

$$
B_{r}=\left\{x \in P C_{1-\gamma}(J, R):\|x\|_{P C_{1-\gamma}} \leq r\right\} .
$$


The operator form is given in Theorem 3.2. The proof is based on the Theorem 2.16. The proof is given in the following steps:

Step 1: The operator $N: B_{r} \rightarrow B_{r}$ is continuous.

Let $x_{n}$ be a sequence such that $x_{n} \rightarrow x$ in $B_{r}$. Then for each $t \in J$, we have

$$
\begin{aligned}
& \left|\left(N x_{n}\right)(t)\left(t-t_{k}\right)^{1-\gamma}-(N x)(t)\left(t-t_{k}\right)^{1-\gamma}\right| \\
& \leq \frac{1}{\Gamma(\gamma)}\left[\sum_{0<t_{k}<t}\left|\psi_{k}\left(x_{n}\left(t_{k}\right)\right)-\psi_{k}\left(x\left(t_{k}\right)\right)\right|\right. \\
& +\sum_{0<t_{k}<t} \lambda I_{t_{k-1}}^{\left(1-\alpha_{1}\right)(1-\beta)+\alpha_{2} \beta}\left|x_{n}\left(t_{k}\right)-x\left(t_{k}\right)\right| \\
& \left.+\sum_{0<t_{k}<t} I_{t_{k-1}}^{1+\beta\left(\alpha_{1}+\alpha_{2}-1\right)}\left|f\left(t_{k}, x_{n}\left(t_{k}\right)\right)-f\left(t_{k}, x\left(t_{k}\right)\right)\right|\right] \\
& +\left(t-t_{k}\right)^{1-\gamma} \lambda I_{t_{k}}^{\alpha_{2}}\left|x_{n}(t)-x(t)\right| \\
& +\left(t-t_{k}\right)^{1-\gamma} I_{t_{k}}^{\alpha_{1}+\alpha_{2}}\left|f\left(t, x_{n}(t)\right)-f(t, x(t))\right| .
\end{aligned}
$$

Since $f$ is continuous, then by the Lebesgue Dominated Convergence Theorem which implies

$$
\left\|\left(N x_{n}\right)(t)-(N x)(t)\right\|_{P C_{1-\gamma}} \rightarrow 0 \text { as } n \rightarrow \infty .
$$

Step 2:The operator $N$ is uniformly bounded.

By Thoerem 3.2 $N\left(B_{r}\right)$ is uniformly bounded. It is clear that $N\left(B_{r}\right) \subset B_{r}$ is bounded.

Step 3:The operator $N$ is equicontinuous. Let $t_{1}, t_{2} \in J, t_{1}>t_{2}$. Then,

$$
\begin{aligned}
& \left|(N x)\left(t_{1}\right)\left(t_{1}-t_{k}\right)^{1-\gamma}-(N x)\left(t_{2}\right)\left(t_{2}-t_{k}\right)^{1-\gamma}\right| \\
& \leq \frac{1}{\Gamma(\gamma)}\left[\sum_{0<t_{k}<t_{1}-t_{2}}\left|\psi_{k}\left(x\left(t_{k}\right)\right)\right|+\sum_{0<t_{k}<t_{1}-t_{2}} \lambda I_{t_{k-1}}^{\left(1-\alpha_{1}\right)(1-\beta)+\alpha_{2} \beta}\left|x\left(t_{k}\right)\right|\right. \\
& \left.\quad+\sum_{0<t_{k}<t_{1}-t_{2}} I_{t_{k-1}}^{1+\beta\left(\alpha_{1}+\alpha_{2}-1\right)}\left|f\left(t_{k}, x\left(t_{k}\right)\right)\right|\right]-\left(t_{1}-t_{k}\right)^{1-\gamma} \lambda I_{t_{k}}^{\alpha_{2}}\left|x\left(t_{1}\right)\right| \\
& +\left(t_{2}-t_{k}\right)^{1-\gamma} \lambda I_{t_{k}}^{\alpha_{2}}\left|x\left(t_{2}\right)\right| \\
& \quad+\left(t_{1}-t_{k}\right)^{1-\gamma} I_{t_{k}}^{\alpha_{1}+\alpha_{2}}\left|f\left(t_{1}, x\left(t_{1}\right)\right)\right|-\left(t_{2}-t_{k}\right)^{1-\gamma} I_{t_{k}}^{\alpha_{1}+\alpha_{2}}\left|f\left(t_{2}, x\left(t_{2}\right)\right)\right| .
\end{aligned}
$$

From Step 1- Lemma 3 combined with Arzela-Ascoli theorem, we conclude that $N$ is continuous and compact. From the application of Theorem 2.16, we deduce that $N$ has a fixed point $x$ which is a solution of the problem Equation (1).

Remark 3.4. Let $z$ is solution of the inequality (2), then $z$ is a solution of the following integral inequality

$$
\mid z(t)-\frac{\left(t-t_{k}\right)^{\gamma-1}}{\Gamma(\gamma)}\left[x_{a}+\sum_{0<t_{k}<t} \psi_{k}\left(z\left(t_{k}\right)\right)-\sum_{0<t_{k}<t} \lambda I_{t_{k-1}}^{\left(1-\alpha_{1}\right)(1-\beta)+\alpha_{2} \beta} z\left(t_{k}\right)\right.
$$




$$
\begin{aligned}
& \left.+\sum_{0<t_{k}<t} I_{t_{k-1}}^{1+\beta\left(\alpha_{1}+\alpha_{2}-1\right)} f\left(t_{k}, z\left(t_{k}\right)\right)\right]+\lambda I_{t_{k}}^{\alpha_{2}} z(t)-I_{t_{k}}^{\alpha_{1}+\alpha_{2}} f(t, z(t)) \mid \\
& \leq \epsilon\left[\frac{m(b-a)^{\gamma-1}}{\Gamma(\gamma)}+\frac{m(b-a)^{\alpha_{1}+\alpha_{2}}}{\Gamma(\gamma) \Gamma\left(2+\beta\left(\alpha_{1}+\alpha_{2}-1\right)\right)}+\frac{(b-a)^{\alpha_{1}+\alpha_{2}}}{\Gamma\left(\alpha_{1}+\alpha_{2}+1\right)}\right] .
\end{aligned}
$$

Theorem 3.5. The assumptions [H1], [H2] and [H3] holds. Then Equation (1) is generalized UHR stable.

Proof. Let $z$ be solution of (4) and by Theorem 3.2 there $x$ is unique solution of the problem

$$
\begin{aligned}
D^{\alpha_{1}, \beta}\left(D^{\alpha_{2}, \beta}+\lambda\right) x(t) & =f(t, x(t)), & & t \in J=[0, T], \\
\left.\Delta I^{1-\gamma} x(t)\right|_{t=t_{k}} & =\psi_{k}\left(x\left(t_{k}\right)\right), & & k=1,2, \ldots, m, \\
I^{1-\gamma} x(a) & =I^{1-\gamma} z(a)=x_{a} . & &
\end{aligned}
$$

Then we have

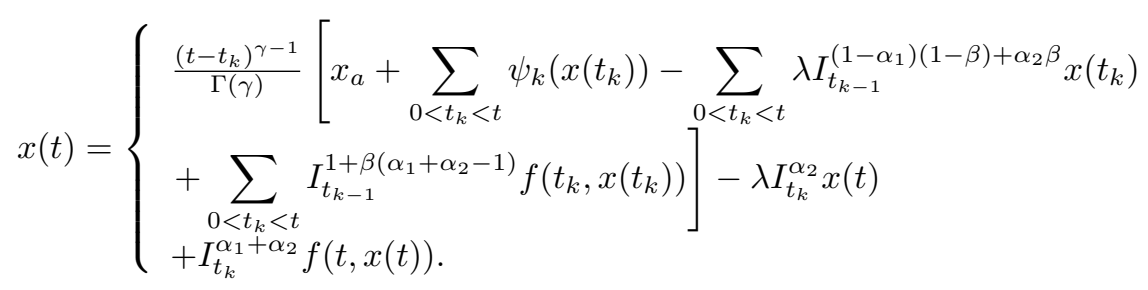

By differentiating inequality (4), for each $t \in\left(t_{k}, t_{k+1}\right]$, we have

$$
\begin{aligned}
& \mid z(t)-\frac{\left(t-t_{k}\right)^{\gamma-1}}{\Gamma(\gamma)}\left[x_{a}+\sum_{0<t_{k}<t} \psi_{k}\left(z\left(t_{k}\right)\right)-\sum_{0<t_{k}<t} \lambda I_{t_{k-1}}^{\left(1-\alpha_{1}\right)(1-\beta)+\alpha_{2} \beta} z\left(t_{k}\right)\right. \\
& \left.+\sum_{0<t_{k}<t} I_{t_{k-1}}^{1+\beta\left(\alpha_{1}+\alpha_{2}-1\right)} f\left(t_{k}, z\left(t_{k}\right)\right)\right]+\lambda I_{t_{k}}^{\alpha_{2}} z(t)-I_{t_{k}}^{\alpha_{1}+\alpha_{2}} f(t, z(t)) \mid \\
& \leq \frac{\left(t-t_{k}\right)^{\gamma-1}}{\Gamma(\gamma)}\left[\sum_{0<t_{k}<t} g_{k}+\sum_{0<t_{k}<t} I_{t_{k-1}}^{1+\beta\left(\alpha_{1}+\alpha_{2}-1\right)} \varphi\left(t_{k}\right)\right]+I_{t_{k}}^{\alpha_{1}+\alpha_{2}} \varphi(t) \\
& \leq\left[\lambda_{\varphi}\left(\frac{m(b-a)^{\gamma-1}}{\Gamma(\gamma)}+1\right)+\frac{m(b-a)^{\gamma-1}}{\Gamma(\gamma)}\right] \varphi(t) .
\end{aligned}
$$

Hence for each $t \in\left(t_{k}, t_{k+1}\right]$, it follows

$$
\begin{aligned}
& |z(t)-x(t)| \\
& \leq \mid z(t)-\frac{\left(t-t_{k}\right)^{\gamma-1}}{\Gamma(\gamma)}\left[x_{a}+\sum_{0<t_{k}<t} \psi_{k}\left(x\left(t_{k}\right)\right)-\sum_{0<t_{k}<t} \lambda I_{t_{k-1}}^{\left(1-\alpha_{1}\right)(1-\beta)+\alpha_{2} \beta} x\left(t_{k}\right)\right. \\
& \left.\quad+\sum_{0<t_{k}<t} I_{t_{k-1}}^{1+\beta\left(\alpha_{1}+\alpha_{2}-1\right)} f\left(t_{k}, x\left(t_{k}\right)\right)\right]+\lambda I_{t_{k}}^{\alpha_{2}} x(t)-I_{t_{k}}^{\alpha_{1}+\alpha_{2}} f(t, x(t)) \mid
\end{aligned}
$$




$$
\begin{aligned}
\leq & \mid z(t)-\frac{\left(t-t_{k}\right)^{\gamma-1}}{\Gamma(\gamma)}\left[x_{a}+\sum_{0<t_{k}<t} \psi_{k}\left(z\left(t_{k}\right)\right)-\sum_{0<t_{k}<t} \lambda I_{t_{k-1}}^{\left(1-\alpha_{1}\right)(1-\beta)+\alpha_{2} \beta} z\left(t_{k}\right)\right. \\
& \left.+\sum_{0<t_{k}<t} I_{t_{k-1}}^{1+\beta\left(\alpha_{1}+\alpha_{2}-1\right)} f\left(t_{k}, z\left(t_{k}\right)\right)\right]+\lambda I_{t_{k}}^{\alpha_{2}} z(t)-I_{t_{k}}^{\alpha_{1}+\alpha_{2}} f(t, z(t)) \mid \\
& +\frac{\left(t-t_{k}\right)^{\gamma-1}}{\Gamma(\gamma)}\left(\sum_{0<t_{k}<t}\left|\psi_{k}\left(x\left(t_{k}\right)\right)-\psi_{k}\left(z\left(t_{k}\right)\right)\right|\right. \\
& +\sum_{0<t_{k}<t} \lambda I_{t_{k-1}}^{\left(1-\alpha_{1}\right)(1-\beta)+\alpha_{2} \beta}\left|x\left(t_{k}\right)-z\left(t_{k}\right)\right| \\
& \left.+\sum_{0<t_{k}<t} I_{t_{k-1}}^{1+\beta\left(\alpha_{1}+\alpha_{2}-1\right)}\left|f\left(t_{k}, x\left(t_{k}\right)\right)-f\left(t_{k}, z\left(t_{k}\right)\right)\right|\right) \\
& +\lambda I_{t_{k}}^{\alpha_{2}}|x(t)-z(t)|+I_{t_{k}}^{\alpha_{1}+\alpha_{2}}|f(t, x(t))-f(t, z(t))| \\
\leq & {\left[\lambda_{\varphi}\left(\frac{m(b-a)^{\gamma-1}}{\Gamma(\gamma)}+1\right)+\frac{m(b-a)^{\gamma-1}}{\Gamma(\gamma)}\right] \varphi(t) } \\
& +\frac{\left(t-t_{k}\right)^{\gamma-1}}{\Gamma(\gamma)}\left(\sum_{0<t_{k}<t} L_{\psi}\left|\left(x\left(t_{k}\right)\right)-\left(z\left(t_{k}\right)\right)\right|\right. \\
& +\sum_{0<t_{k}<t} \lambda I_{t_{k-1}}^{\left(1-\alpha_{1}\right)(1-\beta)+\alpha_{2} \beta}\left|x\left(t_{k}\right)-z\left(t_{k}\right)\right| \\
& \left.+\sum_{0<t_{k}<t} I_{t_{k-1}}^{1+\beta\left(\alpha_{1}+\alpha_{2}-1\right)} L_{f}\left|x\left(t_{k}\right)-z\left(t_{k}\right)\right|\right) \\
& +\lambda I_{t_{k}}^{\alpha_{2}}|x(t)-z(t)|+I_{t_{k}}^{\alpha_{1}+\alpha_{2}} L_{f}|x(t)-z(t)|
\end{aligned}
$$

By Lemma 2.15 there exists a constant $\kappa>0$ independent of $\lambda_{\varphi} \varphi(t)$ such that

$$
|z(t)-x(t)| \leq \kappa \varphi(t) .
$$

Thus, Equation (1) is generalized UHR stable.

\section{Competing Interests}

The authors declare that they have no competing interests.

\section{REFERENCES}

1. Hilfer, R. (1999). Applications of Eractional Calculus in Physics, World scientific, Singapore.

2. Kilbas, A. A. A., Srivastava, H. M., \& Trujillo, J. J. (2006). Theory and applications of fractional differential equations (Vol. 204). Elsevier Science Limited.

3. Podlubny, I.(1999) . Fractional Differential equation, Academic Press, San Diego.

4. Furati, K. M., \& Kassim, M. D. (2012). Existence and uniqueness for a problem involving Hilfer fractional derivative. Computers \& Mathematics with Applications, 64(6), 16161626. 
5. Hilfer, R., Luchko, Y., \& Tomovski, Z. (2009). Operational method for the solution of fractional differential equations with generalized Riemann-Liouville fractional derivatives. Fract. Calc. Appl. Anal., 12(3), 299-318.

6. Vivek, D., Kanagarajan, K., \& Sivasundaram, S. (2016). Dynamics and stability of pantograph equations via Hilfer fractional derivative. Nonlinear Studies, 23(4) ,685-698.

7. Vivek, D., Kanagarajan, K., \& Elsayed, E. M. (2018). Some existence and stability results for Hilfer-fractional implicit differential equations with nonlocal conditions. Mediterranean Journal of Mathematics, 15(1), 15.

8. Vivek, D., Kanagarajan, K., \& Sivasundaram, S. (2017). Dynamics and stability results for Hilfer fractional type thermistor problem. Fractal and Fractional, 1(1), 1-14.

9. Vivek, D., Kanagarajan, K., \& Sivasundaram, S. (2017). Theory and analysis of nonlinear neutral pantograph equations via Hilfer fractional derivative. Nonlinear Studies, 24(3), 699-712.

10. Wang, J., \& Zhang, Y. (2015). Nonlocal initial value problems for differential equations with Hilfer fractional derivative. Applied Mathematics and Computation, 266, 850-859.

11. Beck, C., \& Roepstorff, G. (1987). From dynamical systems to the Langevin equation. Physica A: Statistical Mechanics and its Applications, 145(1-2), 1-14.

12. Fa, K. S. (2007). Fractional Langevin equation and Riemann-Liouville fractional derivative. The European Physical Journal E, 24(2), 139-143.

13. Chen, A., \& Chen, Y. (2011). Existence of solutions to nonlinear Langevin equation involving two fractional orders with boundary value conditions. Boundary Value Problems, 2011(1), 516481.

14. Ahmad, B., Nieto, J. J., Alsaedi, A., \& El-Shahed, M. (2012). A study of nonlinear Langevin equation involving two fractional orders in different intervals. Nonlinear Analysis: Real World Applications, 13(2), 599-606.

15. Harikrishnan, S., Kanagarajan, K., \& Elsayed, E. M. (2018) Existence and stability results for langevin equations with Hilfer fractional derivative, Res. Fixed Point Theory Appl., 10 pages.

16. Baghani, O. (2017). On fractional Langevin equation involving two fractional orders. Communications in Nonlinear Science and Numerical Simulation, 42, 675-681.

17. Yu, T., Deng, K., \& Luo, M. (2014). Existence and uniqueness of solutions of initial value problems for nonlinear langevin equation involving two fractional orders. Communications in Nonlinear Science and Numerical Simulation, 19(6), 1661-1668.

18. Lakshmikantham, V., \& Simeonov, P. S. (1989). Theory of impulsive differential equations (Vol. 6). World scientific.

19. Liu, X., \& Li, Y. (2014). Some antiperiodic boundary value problem for nonlinear fractional impulsive differential equations. In Abstract and Applied Analysis (Vol. 2014). Hindawi.

20. Luo, Z., \& Shen, J. (2006). Global existence results for impusive functional differential equation. J. Math. Anal. Appl., 323(1), 644-653.

21. Ibrahim, R. W. (2012). Ulam-Hyers stability for Cauchy fractional differential equation in the unit disk. In Abstract and Applied Analysis (Vol. 2012). Hindawi.

22. Ibrahim, R. W. (2012). Generalized UlamHyers stability for fractional differential equations. International Journal of mathematics, 23(05), 1250056.

23. Rus, I. A. (2010). Ulam stabilities of ordinary differential equations in a Banach space. Carpathian journal of Mathematics, 103-107.

24. Wang, J., Lv, L., \& Zhou, Y. (2011). Ulam stability and data dependence for fractional differential equations with Caputo derivative. Electronic Journal of Qualitative Theory of Differential Equations, 2011(63), 1-10.

25. Wang, J., \& Li, X. (2015). UlamHyers stability of fractional Langevin equations. Applied Mathematics and Computation, 258, 72-83. 
26. Wang, J., Zhou, Y., \& Fec, M. (2012). Nonlinear impulsive problems for fractional differential equations and Ulam stability. Computers $\&$ Mathematics with Applications, 64(10), 3389-3405.

27. Ye, H., Gao, J., \& Ding, Y. (2007). A generalized Gronwall inequality and its application to a fractional differential equation. Journal of Mathematical Analysis and Applications, 328(2), 1075-1081.

28. Granas, A., \& Dugundji, J. (2013). Fixed point theory. Springer Science \& Business Media.

\section{S. Harikrishnan}

Department of Mathematics, Sri Ramakrishna Mission Vidyalaya College of Arts and Science, Coimbatore-641020, India.

e-mail: hkkhari1@gmail.com

\section{E. M. Elsayed}

Department of Mathematics, Faculty of Science,King Abdulaziz University, Jeddah 21589, Saudi Arabia.

Department of Mathematics, Faculty of Science, Mansoura University, Mansoura 35516, Egypt.

e-mail: emmelsayed@yahoo.com

\section{K. Kanagarajan}

Department of Mathematics, Sri Ramakrishna Mission Vidyalaya College of Arts and Science, Coimbatore-641020, India.

e-mail: kanagarajank@gmail.com 